\title{
Spontaneous Cervical Epidural Hematoma Causing Brown-Sequard Syndrome
}

\author{
Byul Hee Yoon, Ki Seok Park, Sung Sam Jung, Mun Sun Park, Seong-Min Kim, \\ Seung-Young Chung, Jong-Chul Chung, Han-Kyu Kim \\ Department of Neurosurgery, Eulji University College of Medicine, Daejeon, Korea
}

Spontaneous cervical epidural hematoma (SCEH) is a rare clinical entity and has a varied etiology. Urgent surgical decompression should be done to prevent serious permanent neurologic deficits. We describe a 59-year-old female who presented with Brown-Sequard syndrome due to spontaneous cervical epidural hematoma. Initially, she was misdiagnosed as cerebrovascular accident. Cervical magnetic resonance imaging revealed epidural hematoma to the right of the spinal cord extending from C3 to C6. She later underwent surgical evacuation and had complete restoration of neurologic function. The outcome in SCEH is essentially determined by the time taken from onset of the symptom to operation. Therefore, early and precise diagnosis such as careful history taking and MRI evaluation is mandatory.

Key Words: Spontaneous cervical epidural hematoma $\cdot$ Brown-Sequard syndrome $\cdot$ Surgical decompression

\section{INTRODUCTION}

Spontaneous cervical epidural hematoma (SCEH) is a relatively rare disease. Although it generally presents with progressive paralysis or quadriparesis and sensory loss of a symmetric nature, this is not always the case. The implication of delayed diagnosis and treatment of SCEH can be permanent neurological deficit even death. The Brown-Sequard syndrome is an exceptional result of $\mathrm{SCEH}^{7,10)}$. The standard therapy is prompt evacuation of the hematoma. We report a case of spontaneous cervical epidural hematoma presented with a Brown-Sequard syndrome and treated by surgical evacuation.

\section{CASE REPORT}

A 59-year-old female was admitted to the hospital with the symptom of right side weakness. The weakness was developed 6 hours prior to admission and she complained pain on her posterior neck and right shoulder while neck rotation. On

- Received: April 24, 2012 - Revised: September 4, 2012

- Accepted: September 25, 2012

Corresponding Author: Ki Seok Park, MD

Department of Neurosurgery, Eulji University Hospital, 1306 Dunsan-dong,

Seo-gu, Daejeon 302-799, Korea

Tel: +82-42-611-3442, Fax: +82-42-611-3444

Email: ks3432@eulji.ac.kr initial neurologic examination in the emergency room, she had $4 / 5$ motor strength in all the muscle groups of the right upper and lower limbs. Sensation and reflexes were normal. She had taken clopidogrel due to old pontine infaction. Due to hemiparetic symptoms and old infarction history, initially we assumed left cerebral infarction. On initial laboratory data, Platelet count was 331,000 , activated partial thromboplastin time (APTT) was $38.1 \mathrm{sec}$, International normalized ratio (INR) was 1.04. A computed tomography (CT) scan and diffusion magnetic resonance imaging (MRI) of the brain were normal. Evaluating in the ER, the patient's hemiparesis was aggravated to $3 / 5$ motor strength. Sensation was grossly decreased on the right side. Pain perception was decreased on the left side. She underwent an urgent MRI scan of the cervical spine and was found to have an extradural mass posterior and to right of the spinal cord extending from C3 to C6. The mass produced compression and leftward displacement of the cord. The mass demonstrated intermediate signal intensity on T2-weighted image and low signal on T1 (Fig. 1). There were no signs of associated neoplasm or vascular malformations. She was emergently taken to the operating room where she underwent a C3 to C6 hemilaminectomy of right side for hematoma evacuation, confirmed by gross and microscopic inspection. Postoperatively, the patient recovered her sensory symptoms immediately and gradually motor function improved to 4 within the next 24 hours. Postoperatively 14 days, she had complete restoration of her sensory and motor function.

Copyright $₫ 2012$ The Korean Spinal Neurosurgery Society 

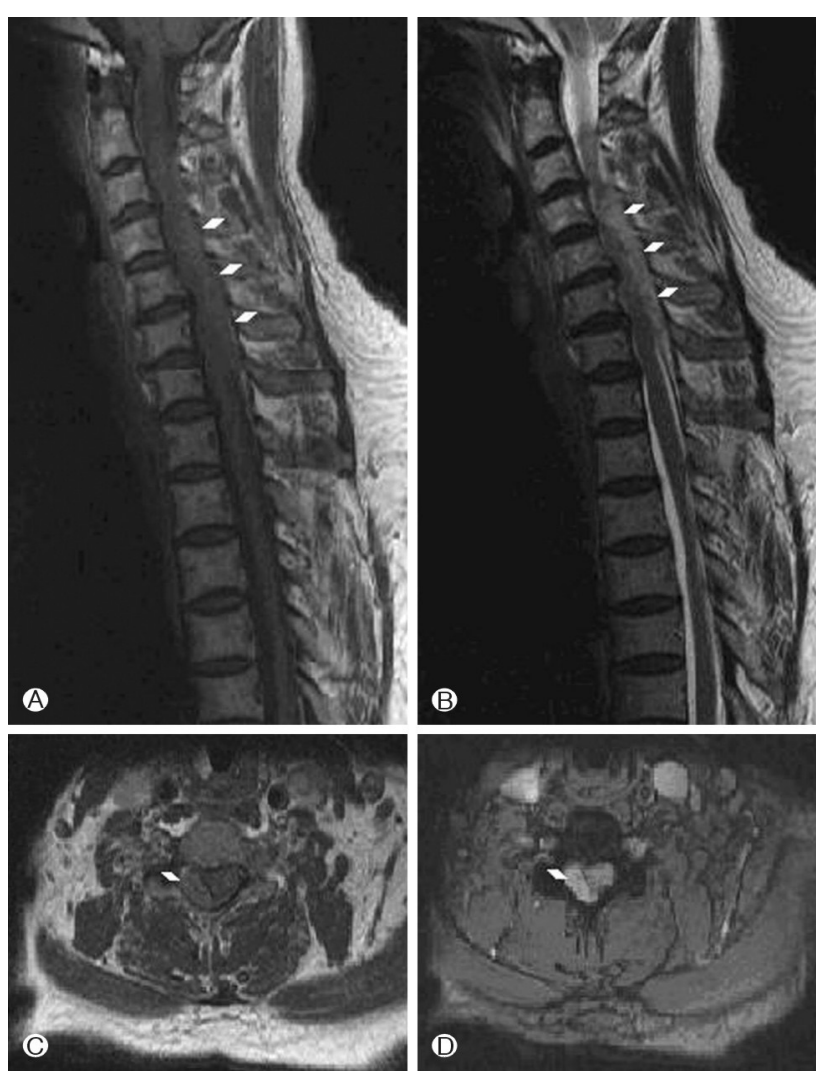

Fig. 1. (A) Preoperative T1 weighted sagittal image-low signal intense mass on beside arrows. (B) Preoperative T2 weighted sagi ttal image-intermediate signal intense mass on beside arrows. (C) Preoperative $\mathrm{Tl}$ weighted axial images-low signal intense mass on beside arrows. (D) Preoperative T2 weigted sagittal imageintermediate signal intense mass on beside arrows

\section{DISCUSSION}

Spontaneous cervical epidural hematoma is a rare but the implication of delayed diagnosis and treatment of spinal epidural hematoma can leave permanent neurological deficit even death ${ }^{3)}$. The neurological outcome was good in those patients that had their hematoma evacuated within 24 hours and the patients with incomplete neurological injury who had a surgical operation performed within 12 hours had an excellent surgical outcome ${ }^{21)}$. Therefore, Patients with mild initial neurological deficit and those with shorter time to operation show better neurological prognosis ${ }^{13)}$. It is still debated whether the etiology of spontaneous epidural hematoma is arterial or venous. Those who support the theory of venous origin claim that epidural veins have thin walls and no valves. As a result of a sudden increase of intra-thoracic and intra-abdominal pressure after valsalva maneuvers like coughing, sneezing or straining, laceration may occur in the venous plexus causing epi- dural hemorrhage ${ }^{6}$. However, because cervical epidural venous pressure is lower than intrathecal pressure, venous bleeding should not be able to compress the dural sac. Therefore, it seems more logical that epidural arteries are the likely causative, especially in light of the compression of the cord that can occur and the posterolateral location of most cervical epidural hematomas ${ }^{4}$. They have explained that the C6 and C7 segments, the most common region of cervical epidural hematomas, were the most mobile cervical segments and extreme compelling movements could therefore cause tearing of these arteries $^{4)}$. Spontaneous cervical epidural hematomas generally present with a sudden onset of neck pain localizing to the site of hematoma. Subsequently (within hours), motor and sensory deficits are observed with paresis or paralysis. Paresis is usually bilateral and rarely unilateral ${ }^{1)}$. Our case had an unusual presentation of spontaneous cervical epidural hematoma with Brown-Sequard syndrome findings of significant motor and sensory involvement of only the half of the body. Pure Brown- Sequard syndrome with complete hemisection of the spinal cord is very rare ${ }^{5)}$. The clinical presentation of incomplete Brown-Sequard syndrome is more frequent, and the neurologic interruption is caused mainly by unilateral involvement of lateral corticospinal tract, dorsal column and ventral spinothalamic tract of the spinal cord $^{14)}$. An extensive review of 613 case studies published between 1826 and 1996 by Kreppel et al. found only 17 reported instances of Brown-Sequard syndrome due to spinal epidural hematoma and thereafter more than six cases reporting Brown-Sequard syndrome due to cervical epidural hematoma ${ }^{10,16,17,18,19,20,22)}$. This unusual presentation can be confused with a cerebrovascular accident which can lead to delayed or misdiagnosis. In our case, initially the patient was misdiagnosed as infarction on left hemisphere and was delayed in diagnosis. Currently, MRI is considered as the first choice of diagnostic method for $\mathrm{SCEH}^{15,23)}$. On T1-weighted images; the signal intensity of the acute hematoma before 48 hours is iso-intense but also may be hyperintense. Subacute and chronic hematomas show hyper-intensity. On T2-wighted images, heterogeneous hyper-intensity to the cord with focal hypo-intensity should suggest the diagnosis of acute spinal epidural hematoma ${ }^{11)}$. Spontaneous cervical epidural hematomas needs emergent surgical decompression especially in cases with neurological deterioration although there were some case reports with spontaneous remission without any surgical treatment ${ }^{8,17}$. But conservative treatment for spontaneous cervical epidural hematoma is not considered unless a patient's neurological deficit resolves spontaneously in the early period soon after onset ${ }^{12)}$. The major factors determining neurological recovery after spontaneous cervical epidural hematoma are the localization on the hematoma (vertebral segments involved), the preoperative neurological con- 
dition, and the operative interval ${ }^{9}$. In complete preoperative sensorimotor loss, surgery within 36 hours correlated with favorable outcome; in incomplete preoperative sensorimotor deficit, favorable outcome correlated with surgery within 48 hours". Our patient had been underwent C3-6 hemilaminectomy and decompression within 24 hours after development of hemiparesis. After operation, a gradual recovery in the neurological status and improvement of sensation was observed following 24 hours. A complete motor and sensory recovery was observed at 1-month follow-up. The common symptom of SCEH is quadriparesis or paraparesis and rarely hemiparesis which can be misdiagnosed as cerebrovascular accident. Early surgery and decompression is a key point in management of SCEH, therefore misdiagnosis can influence the prognosis. Therefore, careful history taking and detailed neurologic examination is indispensable as valuable tools for early diagnosis. Particularly MR imaging is the most reliable investigative procedure and should be accepted as an initial diagnostic tool for all cases of progressive spinal cord dysfunction.

\section{CONCLUSION}

Brown-Sequard syndrome by SCEH is a rare neurosurgical emergency and prompt diagnosis using cervical MRI is very important. But, initially, it can be misdiagnosed as a cerebrovascular accident and treatment can be delayed. It must be kept in mind that the clinical presentation of the cervical epidural hematomas can present as hemiparesis, therefore the patients must be evaluated from this perspective. As seen in our case, immediate and appropriate surgical treatment is essential for a favorable functional neurological recovery in spontaneous cervical epidural hematomas.

\section{REFERENCES}

1. Anderson TJ, Donaldson IM: Spontaneous resolution of cervical spinal epidural haematoma. Postgrad Med J 65:488-490, 1989

2. Awada A, Russell N, AL Fayez N, Naufal R, AL Kohlani H: Spontaneous cervical epidural hematoma: case report. Spinal Cord 36:71-72, 1998

3. Baek BS, Hur JW, Kwon KY, Lee HK: Spontaneous spinal epidural hematoma. J Korean Neurosurg Soc 44:40-42, 2008

4. Beatty RM, Winston KR: Spontaneous cervical epidural hematoma. A consideration of etiology. J Neurosurg 61:143-148, 1984

5. Choi KB, Lee CD, Chung DJ, Lee SH: Cervical disc herniation as a cause of brown-séquard syndrome. J Korean Neurosurg Soc 46:505-510, 2009

6. Correa AV, Beasley BA: Spontaneous cervical epidural hema- toma with complete recovery. Surg Neurol 10:227-228, 1978
7. Egido Herrero JA, Saldanã C, Jiménez A, Vázquez A, Varela de Seijas E, Mata P: Spontaneous cervical epidural hematoma with Brown-Séquard syndrome and spontaneous resolution. Case report. J Neurosurg Sci 36:117-179, 1992

8. Galzio RJ, Zenobii M, D’Ecclesia G: Spontaneous spinal epidural hematoma: report of a case with complete recovery. Surg Neurol 14:263-265, 1980

9. Groen RJ, van Alphen HA: Operative treatment of spontaneous spinal epidural hematomas: a study of the factors determining postoperative outcome. Neurosurgery 39:494-508, 1996

10. Hancock JB, Field EM, Gadam R: Spinal epidural hematoma progressing to Brown-Sequard syndrome: report of a case. J Emerg Med 15:309-312, 1997

11. Holtås S, Heiling M, Lönntoft M: Spontaneous spinal epidural hematoma: findings at $\mathrm{MR}$ imaging and clinical correlation. Radiology 199:409-413, 1996

12. Liu Z, Jiao Q, Xu J, Wang X, Li S, You C: Spontaneous spinal epidural hematoma: analysis of 23 cases. Surg Neurol 69:253260, 2008

13. Mark Fedor, Eric S. Kim, Kai Ding, J. Paul Muizelaar, Kee D. Kim: Spontaneous Spinal Epidural Hematoma: A Retrospective Study on Prognostic Factors and Review of the Literature. Korean J Spine 8(4):272-282, 2011

14. Mastronardi L, Ruggeri A: Cervical disc herniation producing Brown-Sequard syndrome: case report. Spine (Phila Pa 1976) 29:E28-31, 2004

15. Matsumura A, Namikawa T, Hashimoto R, Okamoto T, Yanagida I, Hoshi M, et al: Clinical management for spontaneous spinal epidural hematoma: diagnosis and treatment. Spine J 8:534537, 2008

16. Narberhaus B, Rivas I, Vilalta J, Abós J, Ugarte A: [Transient BrownSéquard syndrome due to spontaneous spinal epidural hematoma]. Neurologia 17:384-387, 2002

17. Neetu R, Chandra MS, Rashmi M: Cervical spinal epidural hematoma with acute Brown-Séquard presentation. Neurol India 54:107-108, 2006

18. Ofluoğlu E, Ozdemir A, Toplamaoğlu H, Sofuoğlu E: Spontaneous cervical epidural hematoma causing Brown-Sequard syndrome: case report. Turk Neurosurg 19:99-102, 2009

19. Riaz S, Jiang H, Fox R, Lavoie M, Mahood JK: Spontaneous spinal epidural hematoma causing Brown-Sequard syndrome: case report and review of the literature. J Emerg Med 33:241-244, 2007

20. Ruiz-Del Barrio I, Antón-Aranda E: [Spontaneous spinal epidural hematoma and Brown-Sequard syndrome]. Rev Neurol 48: 374-375, 2009

21. Shin JJ, Kuh SU, Cho YE: Surgical management of spontaneous spinal epidural hematoma. Eur Spine J 15(6):998-1004, 2006

22. So G, Debata A, Baba S, Tsunoda K, Suyama K, Nagata I: [Spontaneous cervical epidural hematoma presenting with hemiparesis following neck extension: a case report]. No Shinkei Geka 36:731-734, 2008

23. Song KJ, Lee KB: The poor outcome of the delayed diagnosis of acute spontaneous spinal epidural hematoma: two cases report. J Korean Med Sci 20:331-334, 2005 